\section{Alta prevalência de inadequação da ingestão dietética de cálcio e vitamina $D$ em duas coortes de gestantes}

High prevalence of inadequate calcium and vitamin D dietary intake in two cohorts of pregnant women

\author{
Alta prevalencia de inadecuación en la ingestión \\ dietética de calcio y vitamina $D$ en dos \\ cohortes de gestantes
}

\author{
Caroline de Barros Gomes 1 \\ Maíra Barreto Malta 2 \\ José Eduardo Corrente 3 \\ Maria Helena D'Aquino Benício 2 \\ Maria Antonieta de Barros Leite Carvalhaes 1
}

doi: $10.1590 / 0102-311 \times 00127815$

\title{
Resumo
}

Este estudo objetivou identificar a prevalência de inadequação da ingestão, por trimestre, de cálcio e vitamina $D$, em duas coortes de gestantes e fatores correlacionados a esta ingestão. Foram coletados dois recordatórios alimentares de 24 horas em cada trimestre, um relativo a final de semana. Variáveis com correlação significativa com a ingestão desses nutrientes foram incluídas em modelo de regressão linear multivariada, com ajuste por energia. A frequência de inadequação foi estimada pelo método do National Cancer Institute (Estados Unidos). Na coorte A, a inadequação da ingestão de vitamina $D$ não diferiu entre os trimestres; na $B$, houve redução: 99,7\% no 1o para 97,1\% no 3o trimestre. Nas coortes A e B, a inadequação da ingestão de cálcio esteve acima de 70\%, caindo discretamente do 1 o $(89,2 \%$ e 81,4\%) para o 2 o $(79,7$ e 69,1\%) e 3o trimestres $(82,7 \%$ e 72,6\%). Não houve correlação entre as variáveis maternas e a ingestão desses micronutrientes. Conclui-se que há um quadro grave de inadequação da ingestão de vitamina D e cálcio, homogeneamente distribuído entre as gestantes assistidas na rede básica de saúde.

Nutrição Pré-Natal; Gestantes; Consumo de Alimentos; Cálcio; Vitamina D

\footnotetext{
${ }_{1}$ Faculdade de Medicina de Botucatu, Universidade Estadual Paulista "Júlio de Mesquita Filho", Botucatu, Brasil.

2 Faculdade de Saúde Pública, Universidade de São Paulo, São Paulo, Brasil. 3 Instituto de Biociências de Botucatu, Universidade Estadual Paulista "Júlio de Mesquita Filho", Botucatu, Brasil.
}

\author{
Correspondência \\ C. B. Gomes \\ Faculdade de Medicina de \\ Botucatu, Universidade \\ Estadual Paulista "Júlio de \\ Mesquita Filho". \\ Rua Izidoro Bertaglia 970 \\ Botucatu, SP \\ 18610-140, Brasil. \\ carol.bgomes@yahoo.com.br
}




\section{Introdução}

Uma nutrição adequada é de extrema importância em todo o ciclo da vida, com destaque especial ao período gestacional, no qual o estado nutricional da mãe durante a gestação exerce influência sobre os desfechos gestacionais e da saúde infantil 1 . Especificamente quanto aos micronutrientes, a adequação da ingestão de cálcio e vitamina D é particularmente relevante.

Desde 2002 existem evidências de que a baixa ingestão de cálcio está associada ao maior risco de pré-eclâmpsia ${ }^{2}$. Considerando esses resultados e também os subsequentes, a Organização Mundial da Saúde (OMS) passou a recomendar, em áreas com baixa ingestão desse micronutriente e, particularmente para aquelas gestantes com risco elevado para pré-eclâmpsia, a suplementação de 1,5g a 2,0g de cálcio elementar diariamente, da vigésima semana gestacional até o nascimento do bebê ${ }^{3}$. De acordo com a revisão sistemática mais recente, a suplementação de cálcio maior ou igual a 1g/dia está associada com a diminuição significativa do risco de pré-eclâmpsia, especialmente naquelas mulheres com dietas pobres em mineral, reduzindo também o risco de nascimento pré-termo e morte materna ${ }^{4}$. A revisão ainda conclui que, na impossibilidade da prática de altas doses (> $1 \mathrm{~g} /$ dia), a baixa dose de suplementação (500-600mg/ dia) é preferencial à não suplementação, apesar das evidências limitadas na redução do risco de pré-eclâmpsia nesta situação 4 .

Apesar dessas evidências e da recomendação da própria OMS, no Brasil, a suplementação de cálcio profilática não integra o rol de ações preconizadas para a atenção pré-natal dirigida a gestantes de baixo risco obstétrico ou aquele para gestantes com alto risco para pré-eclâmpsia, apesar do reconhecimento de que a suplementação pode ter efeitos favoráveis na prevenção da pré-eclâmpsia 5 . Isso a despeito de a pré-eclâmpsia e eclâmpsia serem as principais causas de morte materna no Brasil no ano de 2013 (17,4\%) (Departamento de Informática do SUS. Óbitos de mulheres em idade fértil e óbitos maternos. http:// tabnet.datasus.gov. $\mathrm{br} / \mathrm{cgi} /$ tabcgi.exe?sim/cnv/mat10uf.def, acessado em 10/Jul/2015) e a segunda no mundo (14\%) 6 , além de já existirem estudos indicando alta prevalência de ingestão insuficiente desse nutriente na gestação.

Um desses estudos foi realizado com dados advindos da Pesquisa de Orçamentos Familiares (POF) brasileira 7 , sem estratificação por trimestre gestacional e não considerando o eventual uso de suplemento: a média de ingestão de cálcio durante a gestação foi de 573,3mg, abaixo da recomendação de $800 \mathrm{mg} /$ dia feita pela Estimated Average Intake (EAR) para gestantes adultas ${ }^{8}$. Outro estudo brasileiro, agora com gestantes da capital paulista, encontrou como média de ingestão dietética diária desse micronutriente $633 \mathrm{mg}{ }^{9}$. Num trabalho internacional, com 1.745 gestantes japonesas, não considerando o uso de suplementos, a média de ingestão foi inferior à dos trabalhos brasileiros: $502,8 \mathrm{mg} 10$, revelando que este não é um problema restrito ao Brasil.

O estado materno para vitamina D também tem sido associado à pré-eclâmpsia, peso e comprimento ao nascer, nascimento pré-termo e diabetes gestacional, com melhores desfechos associados à suficiência da mesma 11,12,13. Contudo, diferentemente do verificado para o cálcio, ainda não existem conclusões claras a respeito da efetividade da suplementação de vitamina D com vistas à redução de desfechos gestacionais negativos 14 .

Há controvérsias sobre a importância da dieta como fonte de vitamina $\mathrm{D}$, na medida em que esta é um pré-hormônio passível de ser sintetizado na pele com a ação dos raios UVB 11. Entretanto, vários autores admitem um papel importante da dieta 12, em especial quando a exposição ao sol é inviável ou desaconselhada. O Institute of Medicine dos Estados Unidos atualizou recentemente as recomendações de ingestão dessa vitamina, sendo $10 \mu \mathrm{g} /$ dia para a população gestante 15 .

Contudo, a ingestão dietética de vitamina D abaixo dessa recomendação tem sido reportada em grávidas de países desenvolvidos. Entre gestantes japonesas, investigando a relação da ingestão de lácteos e vitamina D com sintomas depressivos na gestação, foi encontrada a ingestão dietética diária de 5,7 $\mu$ g desta vitamina 16. Em uma coorte dinamarquesa, com dados dietéticos de 68.447 gestantes, foi encontrada uma média de ingestão

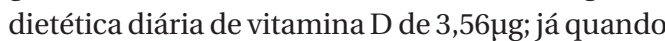
considerada a ingestão de suplementos a média foi de $9,23 \mu \mathrm{g} 17$. Na população gestante brasileira, pelo nosso conhecimento, não foi publicado até o momento nenhum trabalho sobre essa ingestão.

Desse modo, com literatura limitada acerca da situação da ingestão de cálcio e vitamina D por gestantes brasileiras, conhecer a ingestão dietética destes micronutrientes é necessário para subsidiar as recomendações alimentares para esta fase da vida e para a tomada de decisão sobre a necessidade ou não de suplementação profilática.

O objetivo deste trabalho foi estimar a prevalência de inadequação, por trimestre gestacional, da ingestão dietética de cálcio e vitamina $\mathrm{D}$ em duas coortes de gestantes assistidas pela rede pública de atenção primária à saúde, e investigar fatores correlacionados a esta ingestão (idade, escolaridade, nível socioeconômico, trabalhar fora de casa, morar com companheiro, número de partos e estado nutricional pré-gestacional). 


\section{Métodos}

\section{Desenho e população de estudo}

Estudo com duas coortes prospectivas de gestantes, advindas do projeto Impacto de Intervenção para Promoção de Caminhada no Lazer e Alimentação Saudável em Gestantes Atendidas pela Estratégia de Saúde da Família: Um Estudo de Coorte Controlado, desenvolvido com amostra representativa das gestantes que realizaram prénatal no Município de Botucatu, cidade do interior do Estado de São Paulo, Brasil. A coleta de dados compreendeu o período entre novembro de 2012 e fevereiro de 2014. Os detalhamentos do desenho da intervenção, assim como de seus resultados, podem ser encontrados em Malta 18.

As gestantes foram entrevistadas em domicílio e por telefone, em três momentos: primeiro trimestre gestacional $(<14$ semanas gestacionais), segundo trimestre (14-27 semanas) e terceiro trimestre (28-40 semanas). Em cada trimestre foi aplicado um questionário com questões acerca de características socioeconômicas, maternas e sobre a gestação, assim como realizados dois inquéritos alimentares do tipo recordatório de 24 horas (R24h): um presencial e o segundo por telefone, sendo um deles referente a final de semana ou feriado.

Foram incluídas gestantes que estavam matriculadas no pré-natal de baixo risco da rede pública de atenção primária à saúde, com idade igual ou superior a 18 anos, no 1o trimestre de gestação (< 14 semanas). Gemelaridade, presença de doenças ou complicações identificadas no decorrer do estudo, como diabetes, hipertensão, cardiopatia ou qualquer condição adversa que implicasse repouso ou redução de atividade física, foram os critérios de exclusão.

A coorte A iniciou com 181 gestantes assistidas por médicos e enfermeiros de Unidades de Saúde da Família que participaram, previamente, de capacitação para a promoção da alimentação saudável e da caminhada no tempo de lazer em suas consultas de pré-natal 18. No tocante à alimentação saudável, o foco da capacitação foi a promoção do consumo de frutas, verduras e legumes e a redução do consumo de alimentos ultraprocessados 19, principalmente refrigerantes e biscoitos, pelas gestantes; a promoção do consumo adequado de laticínios, importante fonte dos nutrientes investigados no presente estudo, não foi abordada. A coorte B iniciou com 172 gestantes que foram assistidas por profissionais não envolvidos na referida capacitação e que, portanto, receberam a atenção pré-natal habitual.

Das 353 gestantes captadas para as duas coortes e avaliadas quanto ao consumo alimen- tar no primeiro trimestre de gestação, sete se recusaram a continuar participando do estudo; as demais perdas foram decorrentes de mudanças do local de pré-natal (migraram para a assistência privada ou para o pré-natal de alto risco), de cidade de residência e a abortos. Desse modo, da coorte A, foram analisadas 140 e 134 gestantes no segundo e no terceiro trimestres de gestação, respectivamente; da coorte $\mathrm{B}, 141$ e 133 gestantes.

\section{Coleta e avaliação dos dados}

\section{- Caracterização da população}

As variáveis socioeconômicas, demográficas, obstétricas e de estado nutricional das gestantes, coletadas no estudo matriz foram obtidas por questionário presencial, realizado no primeiro trimestre gestacional. Foram utilizadas no presente trabalho as variáveis: idade (18-19 anos, 20|-30, 30|- mais), escolaridade ( $\geq 11$ anos, $8 \mid-11,<8$ anos), nível socioeconômico (classe $\mathrm{B}$, classe $\mathrm{C}$, classes $\mathrm{D} / \mathrm{E}$ ), trabalhar fora de casa (sim ou não), morar com companheiro (sim ou não), número de partos ( 0 , 1 , 2 ou mais), ser da cor branca (sim ou não) e estado nutricional pré-gestacional (baixo peso, eutrofia, sobrepeso, obesidade). O nível socioeconômico foi avaliado de acordo com os critérios de classificação econômica estabelecidos pela Associação Brasileira de Empresas de Pesquisa 20, e o estado nutricional pré-gestacional segundo os critérios da OMS 21.

O primeiro questionário, assim como os demais realizados presencialmente, foi aplicado por entrevistadores treinados para tal atividade, no domicílio da gestante. Nessa primeira entrevista, o projeto foi apresentado à gestante, ela foi convidada a participar da pesquisa e, naquele momento, foi assinado o Termo de Consentimento Livre e Esclarecido.

Após a aplicação de cada questionário foi feita a revisão crítica pelo supervisor de campo e os questionários foram encaminhados para a dupla digitação, que foi realizada no programa EpiData (Epidata Association, Odense, Dinamarca). Na sequência, esse banco foi transcrito para o programa Stata versão 13.0 (StataCorp LP, College Station, Estados Unidos), no qual foi verificada a consistência dos dados.

\section{- Ingestão dietética}

Todos os recordatórios alimentares (um presencial e outro por telefone) foram aplicados usando-se o método Multiple Pass Methods (MPM), pelo qual o entrevistador conduz a entrevista em cinco etapas: listagem rápida, listagem de alimentos comumente esquecidos, definição do horário e refeição, ci- 
clo de detalhamento, revisão final 22. Esse método reduz os erros da medida dietética, pois contribui para que o entrevistado relembre da alimentação do dia anterior e realize o relato detalhadamente 23 . As informações sobre bebidas consumidas foram também coletadas no recordatório.

Para a melhor estimativa do consumo habitual, foi realizado um estrito controle da data de realização dos recordatórios, de modo que a entrevista por telefone ocorreu em dia não consecutivo ao recordatório presencial e em período da semana (sábado/domingo versus dia de semana) distinto. Ou seja, se a realização do recordatório presencial ocorreu em referência a um dia de final de semana ou feriado, o R24h por telefone fez referência a um dia da semana, e vice-versa. Não foram coletadas informações referentes à utilização habitual ou usual de qualquer tipo de suplemento.

Ainda buscando dados de qualidade, na mesma semana em que foram aplicados, os recordatórios eram entregues à supervisão, sendo feita estrita revisão, com ligação telefônica às gestantes para detalhamento de porções, receitas e outras questões, quando necessário.

A estimativa da ingestão habitual de cálcio e vitamina D foi realizada com o software Nutrition Data System for Research (NDSR - University of Minnesota. http://www.ncc.umn.edu/products/), versão 2010. Para a inclusão dos dados dietéticos nesse software foram realizadas padronização e quantificação dos alimentos e preparações, de acordo com as tabelas de Pinheiro et al. 24 e Fisberg $\&$ Villar 25 , que contemplam a grande maioria das preparações consumidas pela população brasileira. Essa etapa permitiu a conversão das medidas caseiras em unidades de peso ou volume (gramas ou mililitros).

Após a digitação de cada recordatório, foi realizada análise de consistência, verificando-se os alimentos e preparações digitados, com especial atenção às unidades de medida e à presença de outliers para porções, pesos, energia e nutrientes. Essas análises foram realizadas com a finalidade de corrigir e minimizar erros e consequentemente a sub ou superestimação do consumo.

\section{Análise de dados}

A prevalência de inadequação de ingestão foi estimada pelo método do National Cancer Institute dos Estados Unidos (NCI) 26 usando-se as rotinas MIXTRAN e DISTRIB 27, elaboradas para o programa SAS forWindows, versão 9.3 (SAS Inst., Cary, Estados Unidos). Esta investigação foi realizada com base em dois recordatórios alimentares de cada trimestre, sendo que o próprio método do NCI, antes da estimativa da prevalência de inadequação, realiza a retirada da variância intrapessoal. Desse modo, o cálculo da prevalência de inadequação foi realizado com uma estimativa da ingestão habitual de todos os indivíduos estudados.

Os valores da EAR (Estimated Average Requirement), paraaingestãodecálcio evitaminaDporgestantes adultas (800mg e 10 $\mathrm{gg}$, respectivamente) 15 , foram utilizados como ponto de corte. A EAR é a estimativa da ingestão média diária de nutrientes necessária para suprir a necessidade da metade dos indivíduos saudáveis em determinado estágio de vida e gênero, sendo o ponto de corte mais adequado para estimar a ocorrência de inadequação da ingestão de grupos populacionais 28 . As diferenças entre as prevalências de inadequação estimadas pelo método do NCI entre os trimestres e entre as coortes foram realizadas utilizando-se o teste de qui-quadrado de tendência (amostras não independentes) e teste de diferenças de proporções (amostras independentes), respectivamente.

A correlação entre as características maternas (idade, escolaridade, nível socioeconômico, trabalhar fora de casa, morar com companheiro, número de partos, ser da cor branca e estado nutricional pré-gestacional) e a ingestão de cálcio e vitamina D foi realizada por coeficiente de correlação de Pearson, também considerando a ingestão média ajustada (retirada da variância intrapessoal) entre os dois recordatórios de cada trimestre, procedimento realizado agora com o auxílio do programa Multiple Source Method (MSM. https://msm dife.de, acessado em 20/Mai/2015). Esse método foi desenvolvido para estimar a ingestão habitual com base em dois ou mais inquéritos alimentares de curto prazo 29.

Pela análise da correlação entre cada variável materna e a ingestão de cálcio e vitamina $\mathrm{D}$, as que apresentaram correlação estatisticamente significativa foram incluídas em modelo de regressão linear multivariada, sendo a variável resposta a ingestão destes micronutrientes com ajuste para energia ${ }^{30}$. Em todos os testes foram considerados $\mathrm{p}<0,05$ como o nível de significância estatística, sendo estas análises realizadas no programa SAS for Windows, versão 9.3.

Este estudo foi aprovado pelo Comitê de Ética da Faculdade de Medicina de Botucatu (CAAE: 32407314.0.0000.5411).

\section{Resultados}

As coortes diferiram quanto às características socioeconômicas: escolaridade ( $p=0,003$ ), classificação socioeconômica $(\mathrm{p}=0,011)$ e trabalho fora de casa $(\mathrm{p}<0,001)$. Ainda assim, nas coortes A e $\mathrm{B}$, respectivamente, predominaram gestantes no estrato de 11 anos ou mais de escolaridade $(43,9 \%$ 
e $55,2 \%)$ e na classe $C(63,6 \%$ e $72,8 \%)$. A maioria das gestantes da coorte A não trabalhava fora de casa $(61,9 \%)$, situação contrária à encontrada na corte $\mathrm{B}$, em que $58,7 \%$ trabalhavam fora. Respectivamente nas coortes $\mathrm{A}$ e B, a maior parte das gestantes morava com companheiro $(73,5 \%$ e $74,4 \%)$, não era primípara $(60,8 \%$ e $54,7 \%)$ e se autorreferia de cor branca (62,9\% e 65,7\%). Quanto ao estado nutricional pré-gestacional, eutrofia foi o mais presente em ambas as coortes, notando-se alta proporção de gestantes com excesso ponderal. A média de idades das gestantes foi de 25,4 \pm 6,1 anos na coorte A e 26,3 $\pm 5,7$ anos na coorte B (Tabela 1).

Considerando-se as coortes A e B juntas, as médias da ingestão energética nos três trimestres gestacionais foram, respectivamente: $1.757 \mathrm{kcal}$, $1.962 \mathrm{kcal}$ e $1.934 \mathrm{kcal}$. As médias de ingestão de vitamina D e cálcio estiveram abaixo dos pontos de corte da EAR (10ug e $800 \mathrm{mg}$, respectivamente)

Tabela 1

Características socioeconômicas, demográficas, obstétricas e de estado nutricional das gestantes segundo coorte. Botucatu, São Paulo, Brasil, 2012-2014.

\begin{tabular}{|c|c|c|c|}
\hline Características & $\begin{array}{c}\text { Coorte A } \\
{[n=181](\%)}\end{array}$ & $\begin{array}{c}\text { Coorte B } \\
{[n=172](\%)}\end{array}$ & Valor de $p$ \\
\hline Idade (anos) & & & 0,282 \\
\hline $18-19$ & 17,7 & 12,2 & \\
\hline $20-30$ & 59,1 & 59,9 & \\
\hline 30 -mais & 23,2 & 27,9 & \\
\hline Escolaridade (anos completos) & & & 0,003 \\
\hline$\geq 11$ & 43,6 & 55,2 & \\
\hline $8-11$ & 28,2 & 29,7 & \\
\hline$<8$ & 28,2 & 15,1 & \\
\hline Classificação socioeconômica * (ABEP) & & & 0,011 \\
\hline B & 8,0 & 11,8 & \\
\hline C & 63,6 & 72,8 & \\
\hline $\mathrm{D} / \mathrm{E}$ & 28,4 & 15,4 & \\
\hline Trabalha fora de casa & & & $<0,001$ \\
\hline Sim & 38,1 & 58,7 & \\
\hline Não & 61,9 & 41,3 & \\
\hline Mora com companheiro & & & 0,841 \\
\hline Sim & 73,5 & 74,4 & \\
\hline Não & 26,5 & 25,6 & \\
\hline Número de partos ** & & & 0,244 \\
\hline 0 & 39,2 & 45,3 & \\
\hline 1 & 26,0 & 29,7 & \\
\hline 2 ou mais partos & 34,8 & 25,0 & \\
\hline Cor branca *** & & & 0,588 \\
\hline Sim & 62,9 & 65,7 & \\
\hline Não & 37,1 & 34,3 & \\
\hline Estado nutricional pré-gestacional \# & & & 0,263 \\
\hline Baixo peso & 4,7 & 5,4 & \\
\hline Eutrofia & 53,2 & 42,5 & \\
\hline Sobrepeso & 25,1 & 32,3 & \\
\hline Obesidade & 17,0 & 19,8 & \\
\hline
\end{tabular}

ABEP: Associação Brasileira de Empresas de Pesquisa.

* Informação perdida sobre poder aquisitivo $n=8$;

** Informação perdida sobre paridade $n=3$;

*** Informação perdida sobre cor da pele $n=3$;

\# Informação perdida sobre índice de massa corporal n $=15$. 
em ambas as coortes e nos três trimestres gestacionais. As coortes não diferiram para a ingestão de vitamina $\mathrm{D}(\mathrm{p}>0,05)$, mas diferiram para a de cálcio nos três trimestres $(\mathrm{p}=0,003 ; \mathrm{p}=0,002$; $\mathrm{p}=0,005$ ), com ingestão maior pelas gestantes da coorte B (Tabela 2).

Na Tabela 2, nota-se que a inadequação do consumo de vitamina $\mathrm{D}$ foi alta nos três trimestres e nas duas coortes. Na coorte $\mathrm{A}$, não houve diferenças significativas entre os trimestres ( $p>0,05)$ : $99,7 \%, 99,0 \%$ e $98,5 \%$; na coorte B, houve discreta redução $(\mathrm{p}=0,008)$ da prevalência de inadequação do primeiro $(99,7 \%)$ para o terceiro trimestres $(97,1 \%)$, sendo que a inadequação no segundo não diferiu do primeiro ou terceiro trimestre $(99,3 \%)$. A inadequação da ingestão de cálcio também foi elevada nas duas coortes, com redução do primeiro $(89,2 \%$ e $81,4 \%$, coortes A e B, respectivamente) para o segundo trimestre $(79,7 \%$ e $68,1 \%$, $(\mathrm{p}<0,001$ e $\mathrm{p}<0,001)$, sendo esta redução mantida no terceiro trimestre $(82,7 \%$ e $72,6 \%, p=0,02$ e $p=0,01)$.

Análises univariadas apontaram várias características correlacionadas à ingestão desses micronutrientes. Na coorte A, a ingestão de vitamina D se correlaciona com: escolaridade, nível socioeconômico, trabalhar fora de casa e cor da pele; a ingestão de cálcio: idade, escolaridade, nível socioeconômico, trabalhar fora de casa, número de partos e estado nutricional pré-gestacional. Na coorte B, escolaridade, nível socioeconômico e estado nutricional pré-gestacional apresentaram corre- lação com a ingestão de vitamina D; escolaridade, nível socioeconômico, morar com companheiro, número de partos e estado nutricional pré-gestacional, à ingestão de cálcio (dados não mostrados em tabelas). Tais variáveis foram incluídas juntas no modelo múltiplo, sendo mostrados na Tabela 3 estes resultados da regressão para a ingestão de cálcio e vitamina D em cada uma das coortes.

Nenhuma das variáveis investigadas (idade, escolaridade, nível socioeconômico, trabalhar fora de casa, presença de companheiro, paridade cor da pele e estado nutricional pré-gestacional), dentro de seu modelo, apresentou correlação independente das demais à ingestão de vitamina $\mathrm{D}$ e cálcio nas duas coortes (Tabela 3).

\section{Discussão}

As prevalências de inadequação da ingestão de cálcio e vitamina $\mathrm{D}$ foram altas em todos os trimestres gestacionais e em ambas as coortes estudadas, com os piores resultados para a vitamina $\mathrm{D}$, com valores sempre acima de $97 \%$. As características maternas socioeconômicas, demográficas e de estado nutricional pré-gestacional não foram determinantes da ingestão dietética de ambos os micronutrientes.

A redução da taxa de inadequação da ingestão de cálcio do primeiro para o segundo e terceiro trimestres, em ambas as coortes, reflete um com-

Tabela 2

Ingestão de vitamina D e cálcio e prevalência de inadequação de ingestão em duas coortes de gestantes por trimestre gestacional. Botucatu, São Paulo, Brasil, 2012-2014.

\begin{tabular}{|c|c|c|c|c|}
\hline \multirow[t]{2}{*}{ Grupo/Trimestre } & \multicolumn{2}{|c|}{ Vitamina D * } & \multicolumn{2}{|c|}{ Cálcio ** } \\
\hline & $\begin{array}{c}\text { Média (DP) } \\
{[\mu \mathrm{g}]}\end{array}$ & $\begin{array}{c}\text { Inadequação } \\
(\%)\end{array}$ & $\begin{array}{c}\text { Média (DP) } \\
{[\mathrm{mg}]}\end{array}$ & $\begin{array}{c}\text { Inadequação } \\
\text { (\%) }\end{array}$ \\
\hline \multicolumn{5}{|l|}{ Coorte A } \\
\hline 1 & $3,2(2,5)$ & 99,7 aA & $544,1(332,9)$ & 89,2 aв \\
\hline 2 & $3,9(2,7)$ & 99,0 aA & $633,3(359,9)$ & 79,7 bB \\
\hline 3 & $4,6(6,1)$ & 98,5 aA & $632,1(338,9)$ & 82,7 bB \\
\hline \multicolumn{5}{|l|}{ Coorte B } \\
\hline 1 & $3,7(2,8)$ & 99,7 aA & $603,5(377,2)$ & 81,4 aA \\
\hline 2 & $4,5(2,8)$ & $99,3 \mathrm{abA}$ & $710,6(362,5)$ & $68,1 \mathrm{bA}$ \\
\hline 3 & $4,6(3,9)$ & $97,1 \mathrm{bA}$ & $690,6(392,4)$ & $72,6 \mathrm{bA}$ \\
\hline
\end{tabular}

Nota: proporções de inadequação seguidas de mesma letra minúscula (fixando coortes, comparando os trimestres) não diferem significativamente pelo teste de diferença de proporções (qui-quadrado de tendência para amostras não independentes). Proporções de inadequação seguidas de mesma letra maiúscula (fixando trimestre) não diferem significativamente pelo teste de diferença de proporções (qui-quadrado para amostras independentes).

* Recomendação pela Estimated Average Intake (EAR) de 10 $\mu \mathrm{g} / \mathrm{dia}$;

** Recomendação pela EAR de $800 \mathrm{mg} /$ dia. 
Ajuste dos modelos de regressão linear múltipla para a ingestão de cálcio e vitamina D, por coorte, de acordo com características maternas. Botucatu, São Paulo, Brasil, 2012-2014.

\begin{tabular}{|c|c|c|c|c|c|c|c|c|c|c|c|c|}
\hline & \multicolumn{6}{|c|}{ Coorte A } & \multicolumn{6}{|c|}{ Coorte B } \\
\hline & \multicolumn{3}{|c|}{ Vitamina D } & \multicolumn{3}{|c|}{ Cálcio } & \multicolumn{3}{|c|}{ Vitamina D } & \multicolumn{3}{|c|}{ Cálcio } \\
\hline & $\boldsymbol{\beta}$ & $\begin{array}{c}\text { Erro } \\
\text { padrão }\end{array}$ & $\begin{array}{l}\text { Valor } \\
\text { de } p\end{array}$ & $\boldsymbol{\beta}$ & $\begin{array}{c}\text { Erro } \\
\text { padrão }\end{array}$ & $\begin{array}{l}\text { Valor } \\
\text { de } p\end{array}$ & $\boldsymbol{\beta}$ & $\begin{array}{c}\text { Erro } \\
\text { padrão }\end{array}$ & $\begin{array}{l}\text { Valor } \\
\text { de } p\end{array}$ & $\boldsymbol{\beta}$ & $\begin{array}{c}\text { Erro } \\
\text { padrão }\end{array}$ & $\begin{array}{l}\text { Valor } \\
\text { de } p\end{array}$ \\
\hline Idade & & & & 1,87 & 1,94 & 0,34 & & & & & & \\
\hline Escolaridade & $-0,20$ & 0,12 & 0,10 & $-14,11$ & 13,21 & 0,28 & $-0,03$ & 0,15 & 0,98 & $-0,22$ & 15,84 & 0,99 \\
\hline Nível socioeconômico & $-0,26$ & 0,18 & 0,88 & $-30,93$ & 18,75 & 0,10 & $-0,09$ & 0,22 & 0,65 & $-10,16$ & 21,6 & 0,64 \\
\hline Trabalhar fora & $-0,15$ & 0,19 & 0,43 & $-17,03$ & 20,25 & 0,40 & & & & & & \\
\hline Morar com companheiro & & & & & & & & & & 16,96 & 24,07 & 0,48 \\
\hline Número de partos & & & & $-3,66$ & 13,51 & 0,78 & & & & $-24,66$ & 13,48 & 0,07 \\
\hline Cor branca & $-0,28$ & 0,19 & 0,15 & & & & & & & & & \\
\hline Estado nutricional pré-gestacional & & & & 0,005 & 1,71 & 0,99 & $-0,03$ & 0,02 & 0,14 & $-2,80$ & 1,92 & 0,14 \\
\hline
\end{tabular}

portamento alimentar característico na gestação: no primeiro trimestre menor consumo alimentar, o segundo como o melhor período, o terceiro trimestre como um período com ligeira redução da ingestão ${ }^{31}$. Essa hipótese pode ser reforçada pelo comportamento da ingestão energética: aumento no segundo trimestre (de $1.757 \mathrm{kcal}$ para $1.962 \mathrm{kcal}$ ) e ligeira redução no terceiro (1.934kcal), reiterando a necessidade do ajuste por energia para a investigação da associação com as variáveis maternas. Contudo, merece destaque que, mesmo com essa redução, as prevalências de inadequação mantiveram-se bastante elevadas em ambas as coortes. Assim, os dados sugerem que a promoção da alimentação saudável, independentemente da prática de suplementação, deve ocorrer desde o início do pré-natal e ser mantida durante toda a gravidez.

Um estudo brasileiro anterior, realizado com dados de amostra nacional, já havia encontrado altas proporções de inadequação da ingestão de cálcio entre gestantes (82\%) 9, valor bastante semelhante aos obtidos no presente trabalho, que incluiu apenas gestantes que realizam o pré-natal na rede pública. Outro estudo brasileiro, com gestantes do interior do Piauí, encontrou inadequação superior a $98 \% 32$.

Prevalências menores de inadequação e maiores médias de ingestão de cálcio na gestação foram reportadas em outros países em desenvolvimento, o que torna a situação brasileira particularmente desfavorável. Entre gestantes tailandesas a prevalência de inadequação foi de $55 \% 33$, bem abaixo da verificada pelo presente estudo; em gestantes do sul da Índia, a média de ingestão diária de cálcio foi de 895mg naquelas diagnosticadas com ane- mia e 797mg nas não anêmicas 34, valores superiores aos encontrados nas gestantes aqui estudadas.

Todavia, tais comparações devem ser vistas com cautela, já que a inadequação da ingestão foi avaliada por métodos distintos, sendo que apenas o estudo com amostra nacional brasileira utilizou o mesmo método do NCI. Entretanto, as disparidades entre países desenvolvidos e em desenvolvimento podem ser verificadas com dois estudos de revisão. Em países desenvolvidos, a ingestão dietética de cálcio durante a gestação nos Estados Unidos, Canadá e Reino Unido e na Europa esteve acima da EAR, a do Japão abaixo e a da Austrália limítrofe em relação a este parâmetro 35 . Já em países de baixa e média rendas, a ingestão de cálcio durante a gestação esteve sempre abaixo ou próxima à EAR 36 .

Assim, considerando os resultados desses estudos sobre ingestão dietética de cálcio durante o período gestacional, a inadequação do consumo de cálcio na gravidez parece refletir o baixo consumo de alimentos fonte deste mineral e não estritamente dependente da utilização ou não de suplementos. No tocante a essa suplementação, um estudo realizado com grávidas de diferentes cidades de regiões brasileiras encontrou apenas $5,1 \%$ delas suplementadas com o micronutriente 37 . Além das evidências de redução do risco de pré-eclâmpsia, nascimento pré-termo e de morte materna severa com a suplementação desse nutriente ${ }^{4}$, também já existem estudos investigando os efeitos da suplementação materna de cálcio em longo prazo: menor índice de cáries aos 12 anos de idade nos filhos de mãe suplementadas com cálcio durante a gestação quando comparados àqueles de mães não suplementadas 38 . A própria OMS, con- 
tudo, ressalta o número limitado de evidências sobre efeitos em longo prazo dessa suplementação 3 . Desse modo, as altas prevalências de inadequação de ingestão dietética de cálcio encontradas no presente trabalho reforçam a necessidade de estudos de intervenção neste sentido, devendo ser consideradas tanto a efetividade de ações educativas para o consumo de alimentos fonte como a suplementação profilática, antes da tomada de decisão pela implantação da suplementação na rotina da atenção pré-natal.

A suplementação de vitamina D durante a gestação também é prática comum em alguns países, apesar de a literatura e da própria OMS ainda não apontarem neste sentido 14,39. Inglaterra, País de Gales, Escócia e Irlanda do Norte 40 , Austrália e Nova Zelândia 41 recomendam essa suplementação em nível nacional. Essas orientações podem ser justificadas dada a baixa exposição solar da população, em virtude da elevada latitude e estações do ano bem definidas, o que reduz a exposição solar. Na pele humana, com a ação da luz ultravioleta o colecalciferol (vitamina D3) é produzido, sendo que tanto a vitamina sintetizada como a obtida por meio da ingestão dietética influenciarão nos níveis séricos da mesma 15.

Apesar de o colégio americano de ginecologia e obstetrícia indicar a suplementação apenas em casos de deficiência ${ }^{42}$, em um estudo observacional com gestantes daquele país a ingestão dietética média foi de $4,81 \mu \mathrm{g} / \mathrm{dia}$, valores ligeiramente superiores aos encontrados em nosso estudo, elevando-se com a suplementação (seja exclusiva do micronutriente ou advinda de uso polivitamínico-mineral) para uma ingestão média de 10,31 $\mu \mathrm{g} /$ dia 12. Em coorte com mais de 60 mil gestantes dinamarquesas, mesmo com suplementação, 66,6\% delas não consumiram essa vitamina adequadamente ${ }^{17}$. Situação semelhante foi observada entre gestantes norueguesas, em que o uso da suplementação, apesar da melhora da ingestão de vitamina $D$, não foi suficiente para atingir as recomendações 43 . Para além da constatada ingestão inadequada em muitos países, uma revisão crítica recentemente publicada concluiu que ainda faltam estudos sobre a relação entre a própria deficiência materna dessa vitamina e os desfechos gestacionais a ela frequentemente associados 44 .

É necessário pontuar que os valores utilizados como referência de ingestão dietética no presente estudo consideram a população americana 15 , cuja exposição solar é distinta de países tropicais, como é o caso do Brasil. Como tratado anteriormente, a exposição solar, com consequente síntese cutânea de vitamina $\mathrm{D}$, pode ser superior àquela considerada como base na recomendação da ingestão e esta divergência, por sua vez, tenha repercussões na interpretação das estimativas de inadequação produzidas. É possível que com maior contribuição da exposição solar a necessidade de ingestão dietética pela população estudada fosse menor Novos trabalhos envolvendo a concomitante avaliação da ingestão, exposição solar e níveis séricos de vitamina $\mathrm{D}$ em gestantes, além dos reflexos de cada um destes nos desfechos da saúde materna e infantil, poderão avançar o conhecimento nesta área e trazer as necessárias evidências para a tomada de decisão sobre intervenções no campo da saúde pública.

Mesmo com essas considerações quanto à vitamina $\mathrm{D}$, a inadequação da ingestão de cálcio e de vitamina $\mathrm{D}$ não parece ser um problema específico das gestantes brasileiras, já que dados da última POF mostram uma alta prevalência de inadequação da ingestão de ambos pela população feminina brasileira com idades entre 19 e 59 anos (90,3\% e 99,6\%, respectivamente) 7 . Essas informações revelam que, apesar de a gestação ser um período particularmente sujeito à inadequação de ingestão de alguns nutrientes ${ }^{8}$, neste caso específico do cálcio e vitamina $\mathrm{D}$, gestantes e mulheres brasileiras em geral estão igualmente expostas à ingestão insuficiente.

No presente estudo, não foi encontrado, após ajuste por energia, qualquer correlação entre a ingestão de cálcio e vitamina $\mathrm{D}$ e características maternas, correlação também não encontrada em gestantes tailandesas e a ingestão de cálcio 33 . Por outro lado, com gestantes de uma das cidades mais pobres dos Estados Unidos (Camden, Nova Jersey), foi encontrada correlação entre a ingestão de vitamina $\mathrm{D}$ com paridade e estado nutricional pré-gravídico, de tal forma que as multíparas e aquelas com baixo peso apresentaram maior ingestão desta vitamina do que nulíparas ou aquelas com excesso de peso ${ }^{12}$. Entre gestantes dinamarquesas foram determinantes para a ingestão suficiente dessa vitamina a idade materna, ser primípara e não fumante 17 .

A ausência dessas correlações no presente estudo deve-se, possivelmente, ao fato de se tratar de uma população bastante homogênea do ponto de vista socioeconômico e cultural: todas as gestantes eram assistidas pela rede pública da atenção primária à saúde do Município de Botucatu. Assim, pode-se afirmar que o quadro de grave inadequação da ingestão de vitamina D e cálcio detectado neste trabalho é homogeneamente distribuído nessa ampla parcela da população que utiliza o Sistema Único de Saúde do município. Mais estudos são necessários, em outros municípios e regiões, no sentido de conhecer melhor a presença (ou não) de inequidades na ingestão de cálcio e vitamina $\mathrm{D}$ em outras populações, uma vez que pode haver diferenças regionais, entre gestantes 
de baixo e alto risco obstétrico e/ou entre níveis socioeconômicos.

Por ora, considerando as evidências e as recomendações atuais da literatura, nossos resultados apoiam a necessidade de ações de educação nutricional com vistas à ingestão adequada de vitamina $\mathrm{D}$ pela dieta. Cabe também o debate sobre qual a exposição solar segura para a gestante, ou seja, frequência, tempo e horário de exposição suficientes para a produção de vitamina D e inócuos para a saúde. Estudos futuros sobre as diversas intervenções nutricionais cabíveis, sem ainda recomendar ou descartar a suplementação profilática de vitamina $\mathrm{D}$ dirigida a gestantes assistidas na rede pública de atenção primária à saúde, podem subsidiar melhor a tomada de decisões sobre o enfrentamento dessa questão.

Avaliar o consumo alimentar é um grande desafio, sendo necessário também considerar as suas limitações inerentes. De acordo com Fisberg et al. 45 , os erros de medida dietética são variados, podendo advir do entrevistado, do entrevistador, do tipo de inquérito utilizado, assim como da análise da informação obtida. Essa análise é dependente da escolha do software, e consequentemente da tabela de composição nutricional, sendo ponto decisivo nesta avaliação, em especial quando se trata da investigação de ingestão de micronutrientes, seja por alimentos faltantes ou por informações falhas e advindas da dificuldade de aferição 46. A própria vitamina D investigada neste estudo tem informação muito limitada sobre seu conteúdo em alimentos, e possivelmente imprecisa, não sendo inclusive contemplada na tabela de composição dos alimentos brasileira 47 . Portanto, estudar o consumo alimentar não é uma tarefa simples, sendo que as dificuldades tornam-se acentuadas durante a gestação com as mudanças alimentares características do período ${ }^{48}$. Possivelmente por esse fato, não é vasta a literatura no sentido de investigação do consumo alimentar na gestação, além da utilização de métodos distintos para as análises de inadequação, limitando a comparação de dados.

A utilização de dois recordatórios alimentares em cada trimestre gestacional fala a favor da qualidade metodológica do presente estudo e apoia a validade de nossos resultados, visto que muitos estudos prévios usaram questionário de frequência alimentar, ferramenta que, no caso da gravidez, não é a mais apropriada 48 e traz uma quantificação menos exata do consumo, influenciada pela habilidade cognitiva do indivíduo para estimar seu consumo médio em longo período de tempo passado 45. Entretanto, é também uma limitação, haja vista que a utilização de no mínimo três recordatórios de 24horas seria mais recomendada para estimar o consumo habitual. Apesar desse fato, acreditamos que a investigação do consumo ao longo da gravidez e com os dois recordatórios de 24 horas acabou por contemplar adequadamente as mudanças alimentares comuns desta fase.

Vale destacar que, apesar das análises terem mantido a separação das gestantes em duas coortes, já que a coorte A foi alvo de uma ação de educação nutricional, a soma das gestantes das coortes A e B constitui amostra representativa das gestantes que realizam pré-natal na rede pública do município pesquisado.

Como os trabalhos nacionais anteriores foram realizados em capitais, uma contribuição deste estudo foi mostrar que a inadequação da ingestão de cálcio e de vitamina D na gestação é problema de saúde pública também em um município de médio porte do interior paulista.

No nosso conhecimento, este é o primeiro estudo brasileiro que avalia e compara a ingestão de cálcio e vitamina D nos três trimestres gestacionais, constatando elevadas proporções de inadequação em toda a gestação, a despeito de ter sido detectada pequena redução da prevalência de inadequação no caso do cálcio, o que reforça a necessidade de intervenções desde o início da atenção pré-natal.

\section{Conclusão}

Foram bastante elevadas as proporções de inadequação da ingestão dietética desses nutrientes em todos os trimestres gestacionais em ambas as coortes, não sendo encontradas correlações entre ingestão e as características maternas: idade, escolaridade, nível socioeconômico, trabalhar fora de casa, presença de companheiro, paridade, cor da pele e estado nutricional pré-gestacional. 


\section{Colaboradores}

C. B. Gomes contribuiu na concepção e projeto, análise e interpretação dos dados, redação do artigo, aprovação final da versão a ser publicada, responsável por todos os aspectos do trabalho na garantia da exatidão e integridade de qualquer parte da obra. M. B. Malta colaborou na concepção e projeto, revisão crítica relevante do conteúdo intelectual, aprovação final da versão a ser publicada, responsável por todos os aspectos do trabalho na garantia da exatidão e integridade de qualquer parte da obra. J. E. Corrente participou na análise e interpretação dos dados, revisão crítica relevante do conteúdo intelectual e aprovação final da versão a ser publicada. M. H. D’A. Benício colaborou na concepção e projeto, revisão crítica relevante do conteúdo intelectual, aprovação final da versão a ser publicada, responsável por todos os aspectos do trabalho na garantia da exatidão e integridade de qualquer parte da obra. M. A. B. L. Carvalhaes colaborou na concepção e projeto, análise e interpretação dos dados, revisão crítica relevante do conteúdo intelectual e aprovação final da versão a ser publicada.

\section{Referências}

1. Barger MK. Maternal nutrition and perinatal outcomes. J Midwifery Womens Health 2010; 55: 502-11.

2. Hofmeyr GJ, Atallah AN, Duley L. Calcium supplementation during pregnancy for preventing hypertensive disorders and related problems. Cochrane Database Syst Rev 2002; (1):CD001059.

3. World Health Organization. Guideline: calcium supplementation in pregnant women. http:// apps.who.int/iris/bitstream/10665/85120/1/ 9789241505376_eng.pdf?ua=1 (acessado em 30/ Mai/2015).

4. Hofmeyr GJ, Lawrie T, Atallah AN, Duley L, Torloni MR. Calcium supplementation during pregnancy for preventing hypertensive disorders and related problems. Cochrane Database Syst Rev 2014; (6):CD001059.

5. Departamento de Atenção Básica, Secretaria de Atenção à Saúde, Ministério da Saúde. Atenção ao pré-natal de baixo risco. http://bvsms.saude. gov.br/bvs/publicacoes/cadernos_atencao_ba sica_32_prenatal.pdf (acessado em 10/Mai/2015).

6. Say L, Chou D, Gemmill A, Tunçalp Ö, Moller AB, Daniels J, et al. Global causes of maternal death: a WHO systematic analysis. Lancet Glob Health 2014; 2:e323-33.

\section{Agradecimentos}

À Unidade de Pesquisa em Saúde Coletiva da Faculdade de Medicina de Botucatu (UPESC) pelo apoio na organização e coleta dos dados. A Thaís Maranhão Negreiros pelo direcionamento e revisão dos dados coletados. Fundação de Amparo à Pesquisa do Estado de São Paulo (FAPESP: 2011/18579-0 e 2014/06865-6).
7. Instituto Brasileiro de Geografia e Estatística. Pesquisa de Orçamentos Familiares 2008-2009: análise do consumo alimentar pessoal no Brasil. Rio de Janeiro: Instituto Brasileiro de Geografia e Estatística; 2011.

8. Dos Santos Q, Sichieri R, Marchioni DM, Verly Junior E. Brazilian pregnant and lactating women do not change their food intake to meet nutritional goals. BMC Pregnancy Childbirth 2014; 14:186.

9. Sato APS, Fujimori E, Szarfarc SC, Borges ALV, Tsunechiro MA. Consumo alimentar e ingestão de ferro de gestantes e mulheres em idade reprodutiva. Rev Latinoam Enferm 2010; 18:113-21.

10. Miyake Y, Tanaka K, Okubo H, Sasaki S, Arakawa M. Intake of dairy products and calcium and prevalence of depressive symptoms during pregnancy in Japan: a cross-sectional study. BJOG 2015; 122:336-43.

11. Mulligan ML, Felton SK, Riek AE, Bernal-Mizrachi C. Implications of vitamin D deficiency in pregnancy and lactation. Am J Obstet Gynecol 2010; 202:429.e1-e429.e9.

12. Scholl TO, Chen X. Vitamin D intake during pregnancy: association with maternal characteristics and infant birth weight. Early Hum Dev 2009; 85:231-4. 
13. Bodnar LM, Simhan HN. Vitamin D may be a link to black-white disparities in adverse birth outcomes. Obstet Gynecol Surv 2010; 65:273-84.

14. De-Regil LM, Palacios C, Lombardo LK, Peña-Rosas JP. Vitamin D supplementation for women during pregnancy. Cochrane Database Syst Rev 2016; (1):CD008873.

15. Institute of Medicine. Dietary reference intakes for calcium and vitamin D. Washington DC: National Academies Press; 2011.

16. Miyake Y, Tanaka K, Okubo H, Sasaki S, Arakawa M. Dietary vitamin D intake and prevalence of depressive symptoms during pregnancy in Japan. Nutrition 2015; 31:160-5.

17. Jensen CB, Petersen SB, Granström C, Maslova E, Mølgaard C, Olsen SF. Sources and determinants of vitamin D intake in Danish pregnant women. Nutrients 2012; 4:259-72.

18. Malta MB. Promoção da caminhada no lazer e alimentação saudável na atenção pré-natal: estudo de intervenção controlado [Tese de Doutorado]. Botucatu: Faculdade de Medicina de Botucatu, Universidade Estadual Paulista "Júlio de Mesquita Filho"; 2015.

19. Departamento de Atenção Básica, Secretaria de Atenção à Saúde, Ministério da Saúde. Guia alimentar para a população brasileira. http://por talsaude.saude.gov.br/images/pdf/2014/novem bro/05/Guia-Alimentar-para-a-pop-brasilieraMiolo-PDF-Internet.pdf (acessado em 15/Dez/ 2015).

20. Associação Brasileira de Empresas de Pesquisa. Critério de classificação econômica Brasil. http:// www.abep.org/ (acessado em 10/Mai/2015).

21. Worl Health Organization. Obesity - preventing and managing the global epidemic: report of a WHO Consultation. Geneva: World Health Organization; 2000. (WHO Technical Report Series, 894).

22. Moshfegh AJ, Rhodes DG, Baer DJ, Murayi T, Clemens JC, Rumpler WV, et al. The US Department of Agriculture Automated Multiple-Pass Method reduces bias in the collection of energy intakes. Am J Clin Nutr 2008; 88:324-32.

23. Raper N, Perloff B, Ingwersen L, Steinfeldt L, Anand J. An overview of USDA's Dietary Intake Data System. J Food Compost Anal 2004; 17:545-55.

24. Pinheiro ABV, Lacerda EMA, Benzecry EH, Gomes MCS, Costa VM. Tabela para avaliação de consumo alimentar em medidas caseiras. 5a Ed. São Paulo: Editora Atheneu; 2008.

25. Fisberg RM, Villar BS. Manual de receitas e medidas caseiras para cálculo de inquéritos alimentares: manual elaborado para auxiliar o processamento de inquéritos alimentares. São Paulo: Signus; 2002.

26. Tooze JA, Midthune D, Dodd KW, Freedman LS, Krebs-Smith SM, Subar AF, et al. A new statistical method for estimating the usual intake of episodically consumed foods with application to their distribution. J Am Diet Assoc 2006; 106:1575-87.

27. National Cancer Institute. Usual dietary inatkes: macros for the NCI method. http://epi.grants.can cer.gov/diet/usualintakes/macros.html? \&url=/ diet/usualintakes/macros.html (acessado em 15/ Dez/2015).
28. Institute of Medicine. Dietary reference intakes: applications in dietary assessment. Washington DC: National Academy of Science; 2000.

29. Haubrock J, Nöthlings U, Volatier JL, Dekkers A, Ocké M, Harttig U, et al. Estimating usual food intake distributions by using the multiple source method in the EPIC-Potsdam Calibration Study. J Nutr 2011; 141:914-20.

30. Willett WC, Howe GR, Kushi LH. Adjustment for total energy intake in epidemiologic studies. Am J Clin Nutr 1997; 65(4 Suppl):1220S-8S.

31. Murphy SP, Abrams BF. Changes in energy intakes during pregnancy and lactation in a national sample of US women. Am J Public Health 1993; 83:1161-3.

32. Lacerda KSSL, Frota KMG, Freire JPA, Voci SM. Prevalência da inadequação no consumo de nutrientes entre gestantes atendidas em unidades básicas de saúde. Rev Bras Promoç Saúde 2014; 27:357-64.

33. Sukchan P, Liabsuetrakul T, Chongsuvivatwong V, Songwathana P, Sornsrivichai V, Kuning M. Inadequacy of nutrients intake among pregnant women in the Deep South of Thailand. BMC Public Health 2010; 10:572.

34. Samuel TM, Thomas T, Finkelstein J, Bosch R, Rajendran R, Virtanen SM, et al. Correlates of anaemia in pregnant urban South Indian women: a possible role of dietary intake of nutrients that inhibit iron absorption. Public Health Nutr 2013; 16:316-24.

35. Blumfield ML, Hure AJ, Macdonald-Wicks L, Smith $\mathrm{R}$, Collins CE. A systematic review and meta-analysis of micronutrient intakes during pregnancy in developed countries. Nutr Rev 2013; 71:118-32.

36. Lee SE, Talegawkar S, Merialdi M, Caulfield LE. Dietary intakes of women during pregnancy in lowand middle-income countries. Public Health Nutr 2012; 16:1340-53.

37. Camargo EB, Moraes LF, Souza CM, Akutsu R, Barreto JM, Silva EM, et al. Survey of calcium supplementation to prevent preeclampsia: the gap between evidence and practice in Brazil. BMC Pregnancy Childbirth 2013; 13:206.

38. Bergel E, Gibbons L, Rasines MG, Luetich A, Belizán JM. Maternal calcium supplementation during pregnancy and dental caries of children at 12 years of age: follow-up of a randomized controlled trial. Acta Obstet Gynecol Scand 2010; 89:1396-402.

39. World Health Organization. Guideline: vitamin D supplementation in pregnant women. Geneva: World Health Organization; 2012.

40. Davies DSC, Jewell T, McBride M, Burns H. Vitamin D - advice on supplements for at risk groups. https://www.gov.uk/government/uploads/system/uploads/attachment_data/file/213703/ dh_132508.pdf (acessado em 15/Jul/2015).

41. The Royal Australian and New Zealand College of Obstetricians and Gynaecologists Board and Council. Vitamin and mineral supplementation and pregnancy. https://www.ranzcog.edu.au/ doc/vitamin-and-mineral-supplementation-inpregnancy.html+\&cd=1\&hl=pt-BR\&ct=clnk\&gl=br (acessado em 15/Jul/2015). 
42. American College of Obstetricians and Gynecologists. Vitamin D: screening and supplementation during pregnancy. Obstet Gynecol 2011; 118:197-8.

43. Haugen M, Brantsæter AL, Alexander J, Meltzer HM. Dietary supplements contribute substantially to the total nutrient intake in pregnant Norwegian women. Ann Nutr Metab 2008; 52:272-80.

44. Weinert LS, Silveiro SP. Maternal-fetal impact of vitamin D deficiency: a critical review. Matern Child Health J 2015; 19:94-101.

45. Fisberg RM, Marchioni DML, Colucci ACA. Avaliação do consumo alimentar e da ingestão de nutrientes na prática clínica. Arq Bras Endocrinol Metabol 2009; 53:617-24

\begin{abstract}
This study aimed to identify the prevalence of inadequate calcium and vitamin D dietary intake and related factors in two cohorts of pregnant women according to trimester of pregnancy. Two 24-hour dietary recall tests were taken in each trimester, one pertaining to weekends. Variables significantly correlated with intake of these nutrients were included in a multivariate linear regression model, adjusted for energy. Prevalence of inadequate intake was estimated according to the National Cancer Institute method (United States). In cohort $A$, inadequate vitamin $D$ did not differ between trimesters; in B there was a reduction: from $99.7 \%$ in the first trimester to $97.1 \%$ in the third. In cohorts $A$ and $B$, inadequate calcium intake exceeded $70 \%$, falling slightly from the first (89.2\% and $81.4 \%$ ) to the second $(79.7 \%$ and $69.1 \%)$ and third trimesters $(82.7 \%$ and $72.6 \%)$. There was no correlation between maternal variables and the intake of these micronutrients. In conclusion, intake of vitamin D and calcium is seriously inadequate and distributed homogeneously among pregnant women in the primary healthcare network.
\end{abstract}

Prenatal Nutrition; Pregnant Women; Food Consumption; Calcium; Vitamin D
46. Anjos LA, Souza DR, Rossato SL. Desafios na medição quantitativa da ingestão alimentar em estudos populacionais. Rev Nutr 2009; 22:151-61.

47. Núcleo de Estudos em Pesquisas em Alimentação. Tabela brasileira de composição dos alimentos. 4a Ed. Campinas: Universidade Estadual de Campinas; 2011.

48. Bertin RL, Parisenti J, Di Pietro PF, Vasconcelos FDAG. Métodos de avaliação do consumo alimentar de gestantes: uma revisão. Rev Bras Saúde Matern Infant 2006; 6:383-90.

\section{Resumen}

Este estudio tuvo como objetivo identificar la prevalencia de inadecuación en la ingestión, por trimestre, de calcio y vitamina $D$, en dos cohortes de gestantes, además de los factores correlacionados con esta inges tión. Se recogieron dos recordatorios alimentarios de 24 horas durante cada trimestre, uno de ellos relativo al fin de semana. Se incluyeron variables en correlación significativa con la ingestión de esos nutrientes, en el modelo de regresión lineal multivariante, con ajuste por energía. La frecuencia de inadecuación fue estimada por el método del National Cancer Institute (Estados Unidos). En la cohorte A, la inadecuación de la ingestión de vitamina $D$ no difirió entre los trimestres; en la B, hubo reducción: un 99,7\% durante el $1^{\circ}$, frente a un $97,1 \%$ en el $3^{\circ}$ trimestre. En las cohortes $A$ y $B$, la inadecuación en la ingestión de calcio estuvo por encima de un $70 \%$, cayendo discretamente del $1^{\circ}$ (89,2\% y 81,4\%), al $2^{\circ}\left(79,7\right.$ y 69,1\%) y $3^{\circ}$ trimestres (82,7\% e 72,6\%). No hubo correlación entre las variables maternas y la ingestión de esos micronutrientes. Se concluye que existe un cuadro grave de inadecuación en la ingestión de vitamina D y calcio, homogéneamente distribuido entre las gestantes asistidas por la red básica de salud.

Nutrición Prenatal; Mujeres Embarazadas;

Consumo de Alimentos; Calcio; Vitamina D
Recebido em 05/Ago/2015

Versão final reapresentada em 07/Mar/2016 Aprovado em 06/Abr/2016 